\title{
Remote-Time Division Multiplexing of Bending Sensors Using a Broadband Light Source
}

\author{
Mikel Bravo and Manuel López-Amo \\ Departamento de Ingeniería Eléctrica y Electrónica, Universidad Pública de Navarra, Campus Arrosadia S/N, \\ Navarra, 31006 Pamplona, Spain \\ Correspondence should be addressed to Mikel Bravo, mikel.bravo@unavarra.es
}

Received 14 February 2012; Accepted 31 July 2012

Academic Editor: Weiqi Jin

Copyright ( 2012 M. Bravo and M. López-Amo. This is an open access article distributed under the Creative Commons Attribution License, which permits unrestricted use, distribution, and reproduction in any medium, provided the original work is properly cited.

This work experimentally demonstrates a remote sensing network which interrogates bending sensors using time-division multiplexing techniques and a broadband light source. The bending sensors are located $50 \mathrm{~km}$ away from the monitoring station. They are based on a simple tie displacement sensor and offer high-resolution measurements of displacement.

\section{Introduction}

Optical fiber intensity sensors based on bend mechanisms have been extensively used because they are simple, cheap, reliable, and can be multiplexed and used simultaneously in multiple locations inside a network. Furthermore, the technique of bending losses has been successfully applied to the measurement of many magnitudes like displacement, pressure, strain, vibration, or temperature [1]. Due to their properties of electromagnetic immunity, the utilization of these sensors has been growing, avoiding serious accidents and guaranteeing a higher level of safety.

Bends in optical fibers cause power propagating in guided modes to be lost by coupling to radiation modes. This type of loss is experienced in fibers wrapped around mandrels or deployed in flexible cables. Attenuations over tens of $\mathrm{dB}$ are possible to be achieved depending on the bending radius in single-mode fibers [2] or fiber tapers [3]. The main objective of the present paper is to report the multiplexing of a macrobending single mode fiber structure to be used as an inexpensive simple sensor, for displacement or high strain measurements.

The sensors used in this work consist of a continuous piece of optical fiber "tied" into the shape of a figure of eight. The sensor was initially demonstrated in [4] using multimode fiber; a singlemode fiber (SMF) version of the sensor was used in [5]. In that paper, 4 sensors were multiplexed using an optical time domain reflectometer (OTDR). Recently, we have used the singlemode version of the sensor to monitoring a concrete beam bending test [6] using also an OTDR. The sensors performed better than fiber Bragg grating (FBG) based sensors when high strain was applied to the concrete beam.

The utilization of an OTDR as the interrogation system of bending sensors, in combination with optical amplifiers, would allow also the remote utilization of them [7].

Remote sensing is related with the continuous monitoring of structures from a central station located tens or hundreds of kilometers away from the field through the critical location of sensors which send information to the central station. This remote capability allows immediate damage detection and consequently necessary actions can be taken.

However, when using an OTDR for remote monitoring of these sensors, this raises two issues:

A low dynamical range of the measurement $(2-3 \mathrm{~dB})$ $[5,7]$ and the cladding mode coupling effects, which causes a nonlinear response in the loss versus the bending radius variations [8]. This occurs when using a single wavelength laser source, as the incorporated one into an OTDR. Total loss of a bent fiber includes the pure bend loss in the bent section and the transition loss caused by the mismatch 


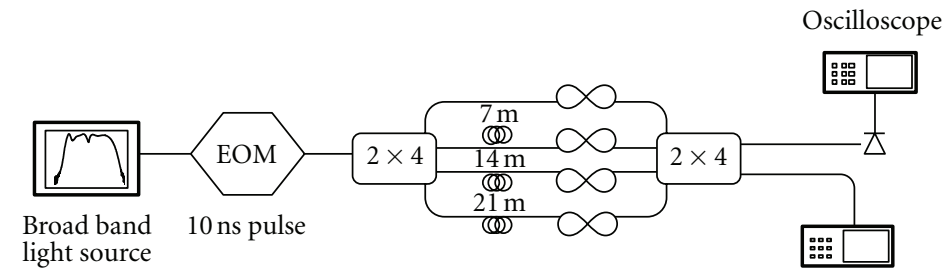

OSA

FIGURE 1: TDM sensing system schematic diagram used for the initial characterization of the sensors.

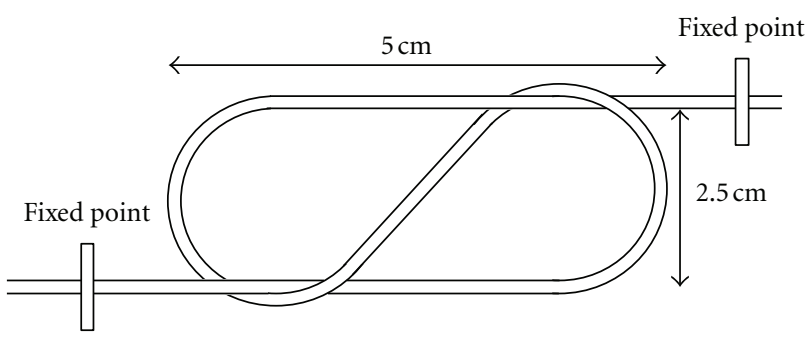

FIGURE 2: Bend tie sensors diagram, including initial dimensions for a nonstressed sensor.

of propagation mode between the bent and the straight sections. For a single mode bent fiber of length $L$, the pure bend loss can be calculated by

$$
L_{s}=10 \log _{10}(\exp (2 \alpha L))=8.686 \alpha L,
$$

where $\alpha$ is the so-called bend loss coefficient, which is determined by the fiber structure, bending radius, and wavelength of the light. The existence of the coating layer will produce a so-called whispering gallery mode for a bent fiber, due to the reflection of the radiated field at the interface between the cladding layer and the coating layer. In order to consider the effect of this reflection on the bend loss, more complicated formulas for the bend loss coefficient $\alpha$ have been developed [9] which justifies the power oscillation.

Because these oscillating peaks change with wavelength, a straightforward way of soften their effect is to use a broadband light source instead the used in conventional OTDRs.

In this work, we demonstrate the advantages of the utilization of a broadband light source for time division multiplexing (TDM) of remote bend loss sensors, increasing the resolution of the measurements and avoiding the dispersion problems caused by the light source.

\section{Methodology}

The initial developed system for TDM multiplexing of bend loss sensors is depicted in Figure 1.

The sensing section consists of four intensity sensors arranged in a parallel (star) configuration. The sensors are based on the bend tie sensor diagram firstly proposed by Sienkiewicz and Shukla (Figure 2) [4]. They are capable of measuring large displacements and consequently high deformations when their ends are attached to a concrete

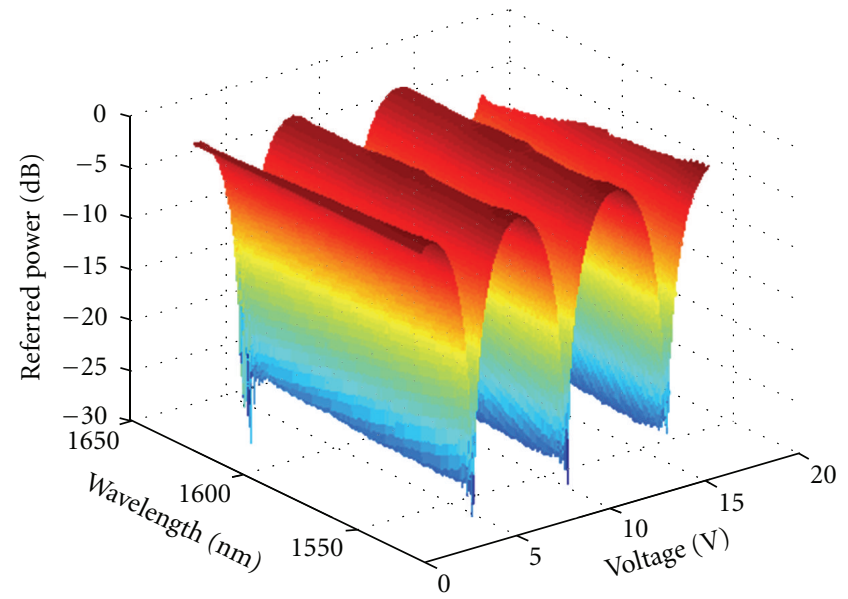

FIGURE 3: EOM response to the biasing point variation.

beam, for example [6]. In order to characterize these sensors, their input and output fiber sections (described as "fixed point" in Figure 2) are placed on two high precision motorized stages which makes that the tie shape changes with the displacement. Therefore, there is a curvature change which increases or decreases the losses. These stages provide a minimum displacement of $1.7 \mathrm{~nm}$. For this work, we have developed a LabVIEW program which integrates all the instruments to make measurements of the displacement of the sensors each $3.4 \mathrm{~mm}$. Also the fiber of the tie sensor is smeared of lithium grease for achieving a soft sensor glide and a good repeatability.

This characterization setup allows us to make a highresolution characterization and to see the cladding mode effects that are present in the most part of the bending sensors systems.

In order to multiplex the sensors by using TDM techniques in a star configuration, two $2 \times 4$ couplers are used to divide the launched signal and to collect the intensity modulated outputs. To discriminate the sensors, different fiber delays are included in each sensing branch.

The characterization system includes a broad band light source whose spectrum is depicted in Figure 5, before and after the modulator, an EOM (electro-optic modulator), an optical spectrum analyzer (OSA), and an oscilloscope.

The operation mode is simple: a broad band light pulse of $10 \mathrm{~ns}$ enters into the first $2 \times 4$ coupler which divides this pulse in 4 almost identical pulses travelling through different 


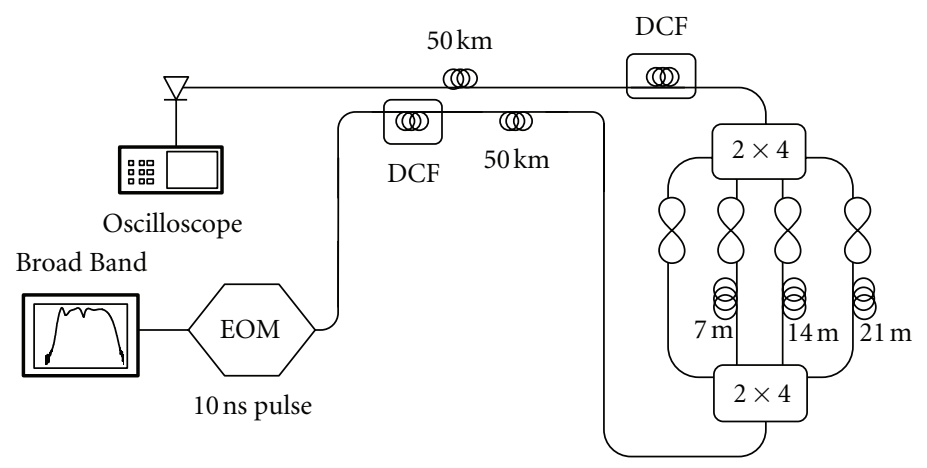

FIgURE 4: Remote $(50 \mathrm{~km})$ sensor system.

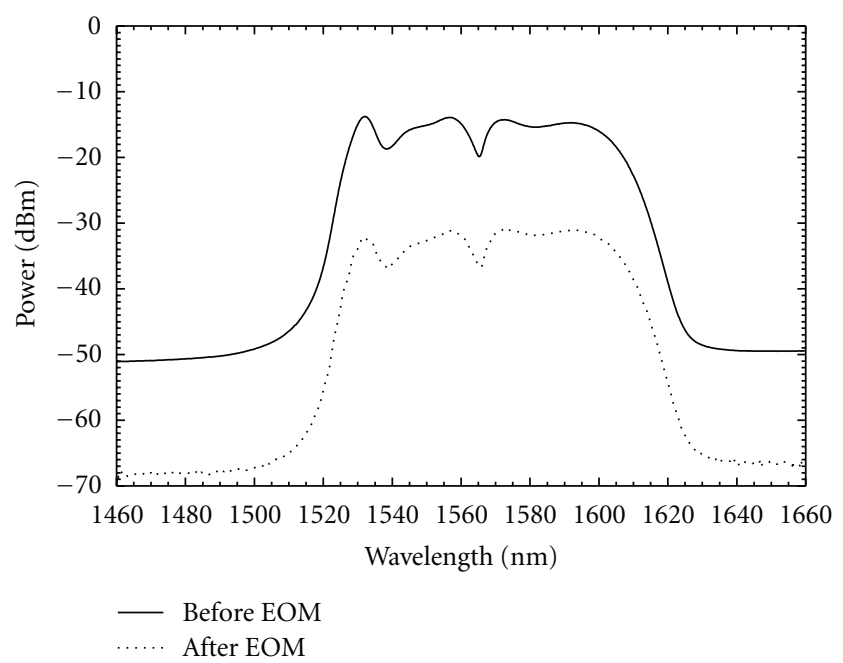

FIGURE 5: Broadband light source spectrum measured before and after the EOM.

fiber delay lines before being attenuated by the sensors, these sensors change their behavior while the motorized stages modify the distance between the fixed points. Afterwards, the pulses are recoupled in the second $2 \times 4$ coupler. Finally, four different pulses corresponding to the different sensors are detected by a New Focus 1811, $125 \mathrm{MHz}$, and 900-1700 nm high sensitivity detector. This detector shows a high trans impedance gain $(4 \times 104 \mathrm{~V} / \mathrm{A})$ and a low noise equivalent power (NEP), having a 3 ns rise time. Finally, the electrical signal offered by the detector is monitored by an oscilloscope. The high sensitivity characteristic of the detector is due to the low optical power present at the end of the remote network. The OSA was used to characterize the sensors spectral behavior using the spare output port of the $2 \times 4$ coupler.

We use a broadband light source from Nettest, model fiberwhite SPL, which covers a wavelength spectrum from 1525.5 to $1611.7 \mathrm{~nm}$, with a maximum output power of $18.8 \mathrm{dBm}$ and power stability of $0.005 \mathrm{~dB}$. This broadband source avoids the oscillations measured in the curvature loss function because of the cladding mode coupling process when a narrow band laser is used [7]. The cladding mode excitation has a wavelength displacement according to the bending radius. Therefore, the losses caused by curvatures are very dependent on the wavelength. By using the broad band source, we achieve a loss averaging of this dependence, avoiding the unwanted effect of the cladding mode recoupling. Because of the nonuniform spectrum of the broadband source, we have referenced the measurements (Figures 3 and 7) regarding the first spectrum obtained with a nonstressed sensor. On the other hand, the utilization of short bending radius loops as mode scramblers, as we did in previous works $[5,6]$, contributes to attenuate the excited cladding modes improving also the accuracy of the measurements. These fiber loops were optimized for each sensor. The loops configuration was developed after several experimental tests, showing the optimum cladding mode suppression behavior. In our experimental set-up, we used only one loop before two of the sensors (the second and the fourth sensor).

$\mathrm{A} \mathrm{LiNbO}_{3}$ electro-optical "UTP APETM $2 \times 2$ interferometric switch" acts as an electro-optic amplitude modulator and it is used to generate short pulses from the light source. The EOM transfer function and its biasing point are two crucial parameters to be controlled. Figure 3 shows the characterization of the modulator response to the changes of the biasing point. These measurements were made by modulating the broad band spectrum, which is registered 


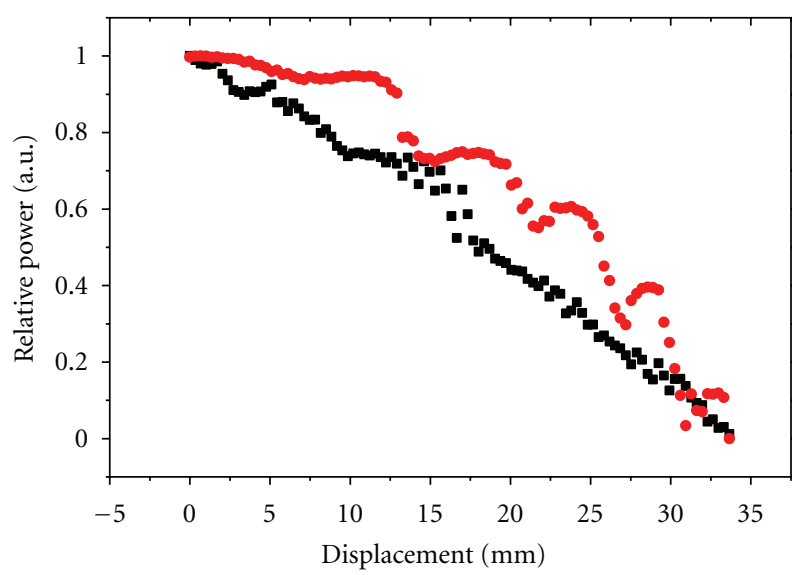

- Broad band

- Narrow band

Figure 6: Measured transfer function of a displacement sensor when a laser diode (red) and a broadband light source (black) are used.

by an OSA. A computer controlled DC source increased $0.01 \mathrm{~V}$ the biasing point in each measurement. This $3 \mathrm{D}$ graph shows the extinction ratio versus the wavelength and the polarization voltage applied. This polarization point is a crucial parameter to be controlled and to be stabilized. The transfer function of the EOM modifies the spectrum of the source while the biasing point controls the amplitude of the pulses.

In order to check the application of this structure for remote sensing, we introduced two $50 \mathrm{~km}$ fiber reels between the light source and the detector, as shown in Figure 4.

Dispersion is a crucial aspect to be taken into account when the optical path is enlarged and a modulated broadband light source is used. Because of this reason, two long dispersion compensation fiber (DCF) sections are used to compensate the dispersion caused by the $100 \mathrm{~km}$ of standard single mode fiber (SMF28). From our measurements we have obtained that the DCF reduces correctly the dispersion, although we have used a broadband source, allowing the sensors detection. However, the price to pay is an additional $7.8 \mathrm{~dB}$ loss.

\section{Results}

This section presents the experimental results for the proposed system. Firstly, we have compared the behavior of one of our displacement sensors using a laser diode from an OTDR (EXFO FTB-7423B-B) and the light from our modulated broadband light source. Figure 5 shows the broad band source spectrum both before and after the EOM. As it is explained before, the biasing point of the EOM is a crucial parameter to be controlled and the biasing point in this case is placed at the minimum point level of the EOM response for achieving the best dynamic range of the modulated pulses.

Figure 6 shows the characterization of one of our intensity sensors when displacement is applied and the two

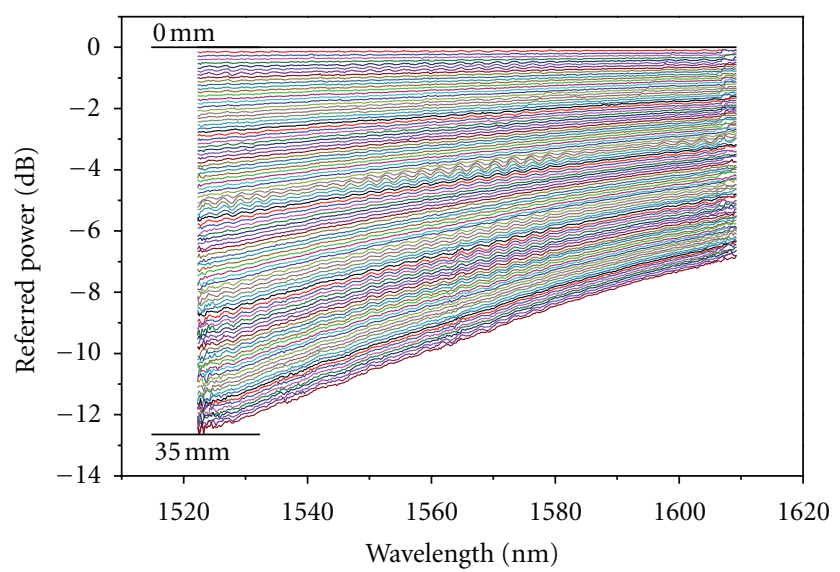

FIGURE 7: Spectrum evolution according the applied displacement after the sensor (the displacement applied is marked inside).

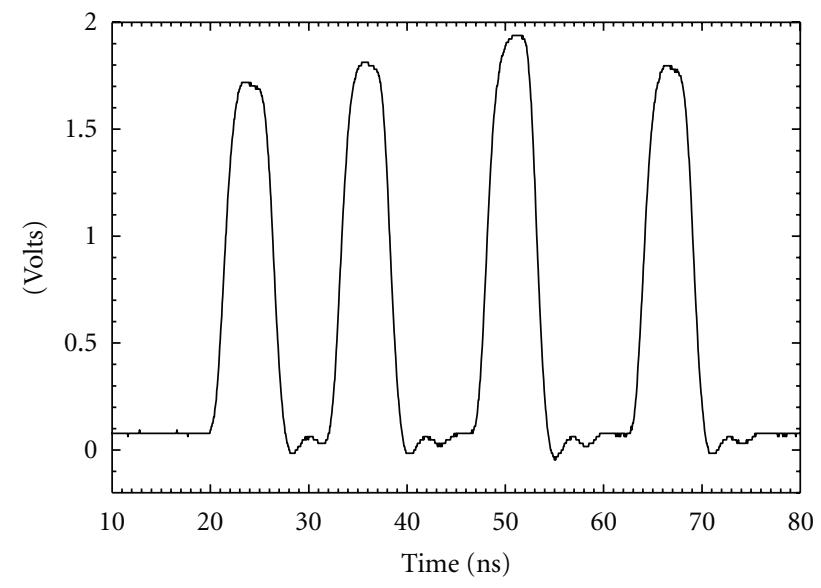

FIGURE 8: Oscilloscope view of the four sensors' received pulses.

different light sources are used. The red trace corresponds to the measurement of the sensor when the OTDR laser is used in a transmissive configuration, as we did in [6]. A nonlinear behavior as in [8] is observed. This behavior limits the resolution and applicability of the sensor. The nonlinear behavior corresponds to the excitation of new cladding modes when the bending radius is reduced with the applied displacement, and this excitation shifts with wavelength [8]. Thus, when a broadband light source is used and all its spectral components are simultaneously introduced into the bending sensor, an average effect is obtained and the linearity of the transfer function is improved.

Figure 7 shows the evolution of the attenuation spectra as the bending radius changes. The first trace (corresponding to the shown in Figure 5, "After EOM") is taken as the reference one. Thus, to calculate the attenuation, the trace taken in each bending state is subtracted to the reference one. The results confirm the dependence of macrobendings with the wavelength.

Figure 8 shows the received signal from the 4 bend tie multiplexed sensors using a high sensitivity detector. For the 


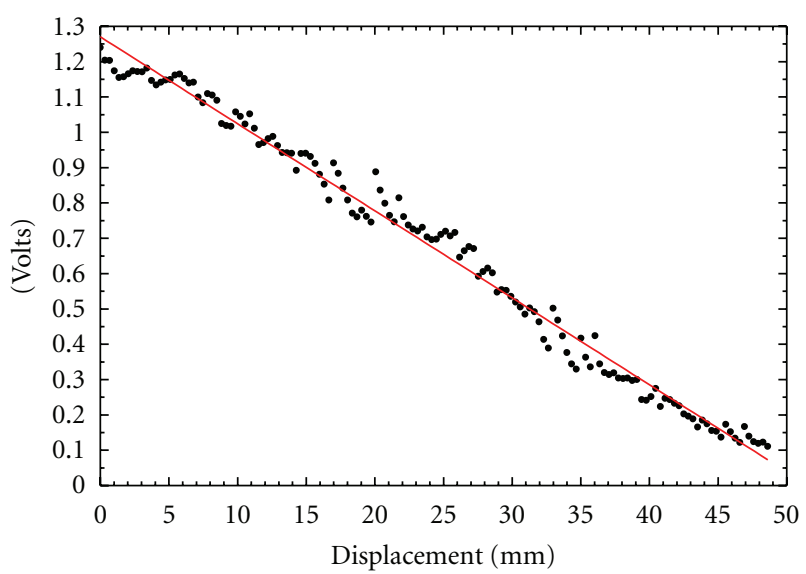

FIGURE 9: First sensor received signal evolution when displacement is applied.

experiment, we have tested each sensor separately and we have checked the crosstalk for the other sensors. A delay of approximately $20 \mathrm{~ns}$ (corresponding to a $7 \mathrm{~m}$ long fiber coil) has been applied to the pulse corresponding to this sensor. The number of sensors that can be multiplexed using this scheme is limited by the received optical power from each sensor. Theoretically, in a nonremote setup is possible to multiplex up to 32 sensors using our topology and equipments. However, for remote applications, this number should be reduced if a good received SNR is desired maintaining the same resolution. Figure 9 shows the evolution of the first sensor measured signal at the detector as it is displaced. In this result, we have a linear response and the cladding mode coupling effect does not appear. A linear fit was performed in the graph to show the linear response of the sensor.

Figure 10 shows the measured displacement behavior of the four sensors. The received signal levels are different because of the different mode scrambler loops calibration parameter explained before.

An important parameter to study is the crosstalk between sensors. In this work, the crosstalk was checked for each sensor. Figure 11 shows the second sensor behavior and the simultaneous signal variations obtained from the others. This figure demonstrates the crosstalk free behavior of this system because the received pulses corresponding to each sensor has its own time slot without suffering overlapping. Therefore, the size of the pulse and the delay of each branch must be well controlled to prevent pulse overlapping.

We have also made an identical characterization of the sensors using the remote sensor system configuration which has the sensors $50 \mathrm{~km}$ away from the monitoring equipments. On this occasion (Figure 12), the evolution of the sensor voltage, as the sensor is displaced, is as linear as the previous one but the dynamic range in this case is much lower. However, it is still sufficient to perform the desired measurements.

Finally, the system resolution, accuracy, and repeatability have been tested for both systems, local and remote systems.

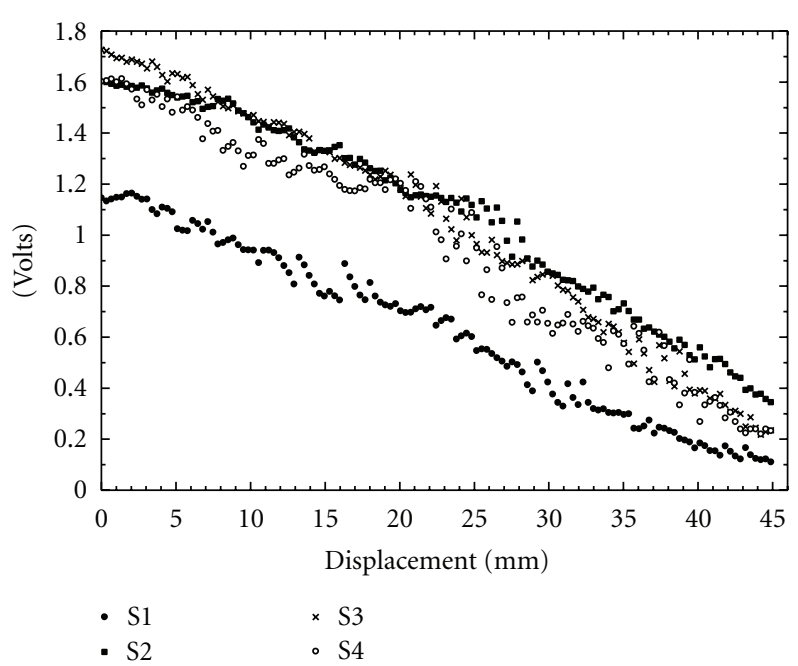

FIGURE 10: All sensor received signals evolution when displacement is applied.

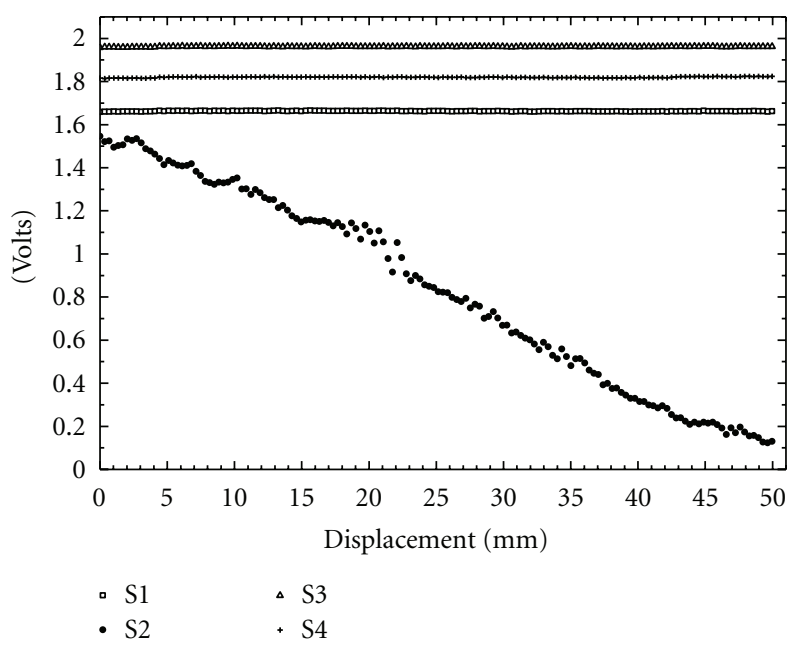

FIGURE 11: Sensors "S1, S3 and S4" crosstalk behavior when displacement is applied to "S2".

Figure 13 shows different measurements along time. With this aim, 30 measurements per minute have been taken without any sensor displacement. The aim of these measurements is to check the system noise and the measurement error versus time. The light source and the utilized photodetector will introduce the corresponding intensity noise and quantum and thermal noise, In order to reduce this noise, averaging of the received signals was used. Other possible measurement errors, coming from instabilities from the EOM and sensor network instabilities may be reduced using an intensity referenced scheme [10]. Using these data, we can estimate the system resolution and accuracy. The measurement frequency can be as fast as $1 \mathrm{~Hz}$, being the real limitation the oscilloscope acquisition speed and data processing time.

The nonremote sensor system resolution is $\pm 0.5 \mathrm{~mm}$ and the remote sensor configuration resolution is $\pm 1 \mathrm{~mm}$ in the measurement range of $35 \mathrm{~mm}$. These measurements were 


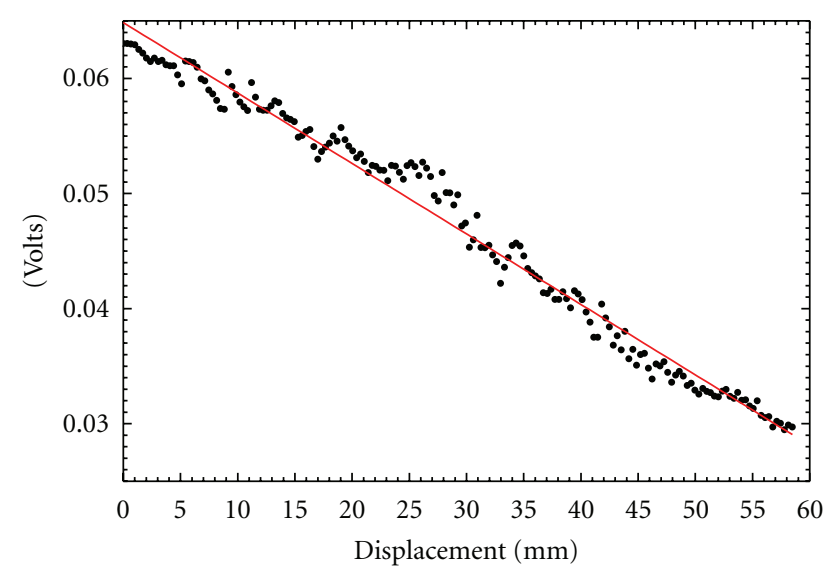

FIGURE 12: Remote sensor detected signal evolution versus displacement.

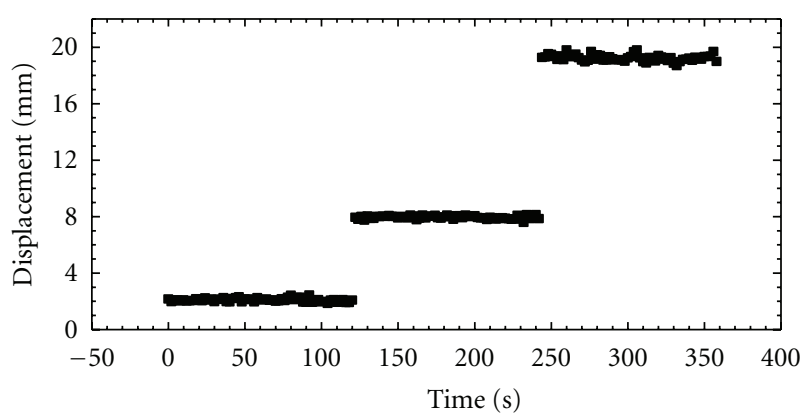

(a)

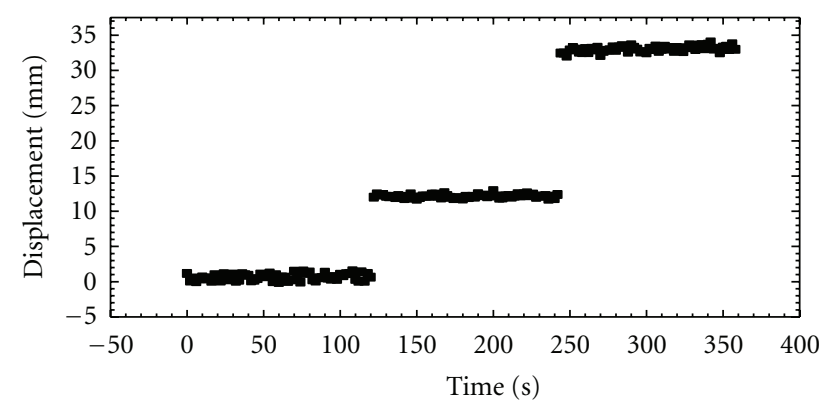

(b)

Figure 13: Local (a) and remote (b) measurements for resolution calculations.

obtained making an average of 64 oscilloscope traces in order to reduce noise. The measured repeatability, defined as the maximum difference between the consecutive readings of the sensors [11], was $\pm 1.14 \%$.

\section{Conclusion}

The utilization of broadband light sources for time-division multiplexing of bend sensors has been demonstrated. An in-depth study of the cladding-mode coupling effects has been carried out to validate the proposed solutions and to improve the sensing characteristics. The adopted light source improves the resolution and the linear behavior of the measurements. We have also demonstrated that these sources can also be used in remote sensing, multiplexing 4 bend loss sensors for displacement measurements $50 \mathrm{~km}$ away from the header of the system, without the utilization of optical amplifiers.

\section{Acknowledgments}

Thanks are due to Montserrat Fernández-Vallejo for her help in the paper preparation and useful discussions, and the authors are grateful to the Spanish Government Project TEC2010-20224-C02-01.

\section{References}

[1] J. M. López-Higuera, "Introduction to fibre optic sensing technology," in Handbook of Optical Fibre Sensing Technology, pp. 1-23, 2002.

[2] W. A. Gambling, H. Matsumura, C. M. Ragdale, and R. A. Sammut, "Measurement of radiation loss in curved singlemode fibres," Microwaves Optics and Acoustics, vol. 2, no. 4, pp. 134-140, 1978.

[3] F. J. Arregui, I. R. Matías, C. Bariain, and M. López-Amo, "Experimental design rules for implementing biconically tapered single mode optical fibre displacement sensors," in European Workshop on Optical Fibre Sensors, vol. 3483 of Proceedings of SPIE, pp. 164-168, July 1998.

[4] F. Sienkiewicz and A. Shukla, "A simple fiber-optic sensor for use over a large displacement range," Optics and Lasers in Engineering, vol. 28, no. 4, pp. 293-304, 1997.

[5] N. M. P. Pinto, O. Frazão, J. M. Baptista, and J. L. Santos, "Quasi-distributed displacement sensor for structural monitoring using a commercial OTDR," Optics and Lasers in Engineering, vol. 44, no. 8, pp. 771-778, 2006.

[6] M. Bravo, J. Sáenz, M. Bravo-Navas, and M. Lopez-Amo, "Fiber optic sensors for monitoring a concrete beam high strain bending test," Journal of Lightwave Technology, vol. 30, no. 8, pp. 1085-1089, 2012.

[7] M. Bravo, M. Fernandez-Vallejo, and M. Lopez-Amo, "Hybrid OTDR-fiber laser system for remote sensor multiplexing," IEEE Sensors Journal, vol. 12, no. 1, pp. 174-178, 2012.

[8] H. Renner, "Bending losses of coated single-mode fibers: a simple approach," Journal of Lightwave Technology, vol. 10, no. 5, pp. 544-551, 1992.

[9] Q. Wang, G. Farrell, and T. Freir, "Theoretical and experimental investigations of macro-bend losses for standard single mode fibers," Optics Express, vol. 13, no. 12, pp. 4476-4484, 2005.

[10] M. Bravo, A. M. R. Pinto, M. López-Amo, J. Kobelke, and K. Schuster, "High precision micro-displacement fiber sensor through a suspended-core Sagnac interferometer," Optics Letters, vol. 37, no. 2, pp. 202-204, 2012.

[11] D. S. Nyce, Linear Position Sensor. Theory and Application, John Wiley \& Sons, 2004. 

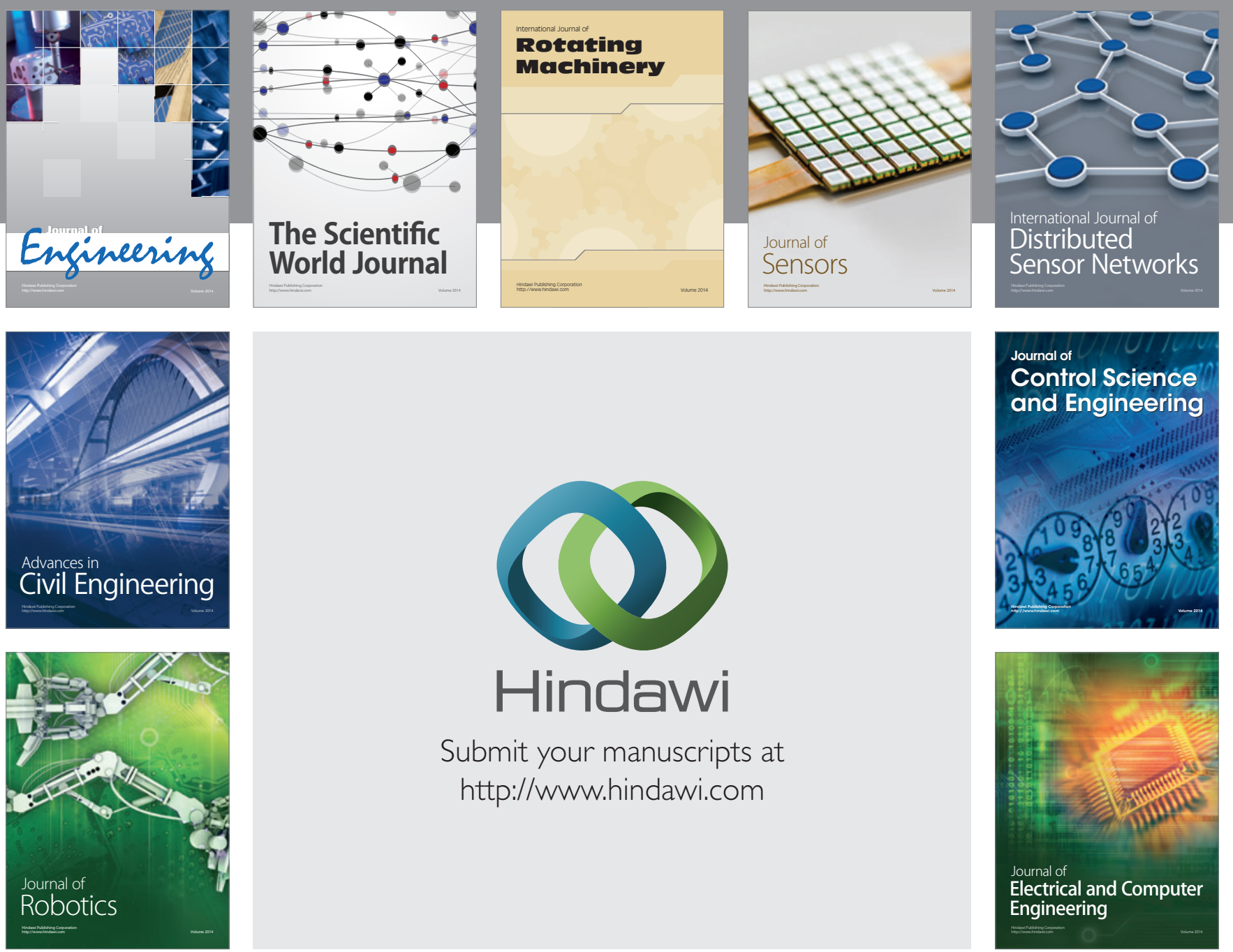

Submit your manuscripts at

http://www.hindawi.com
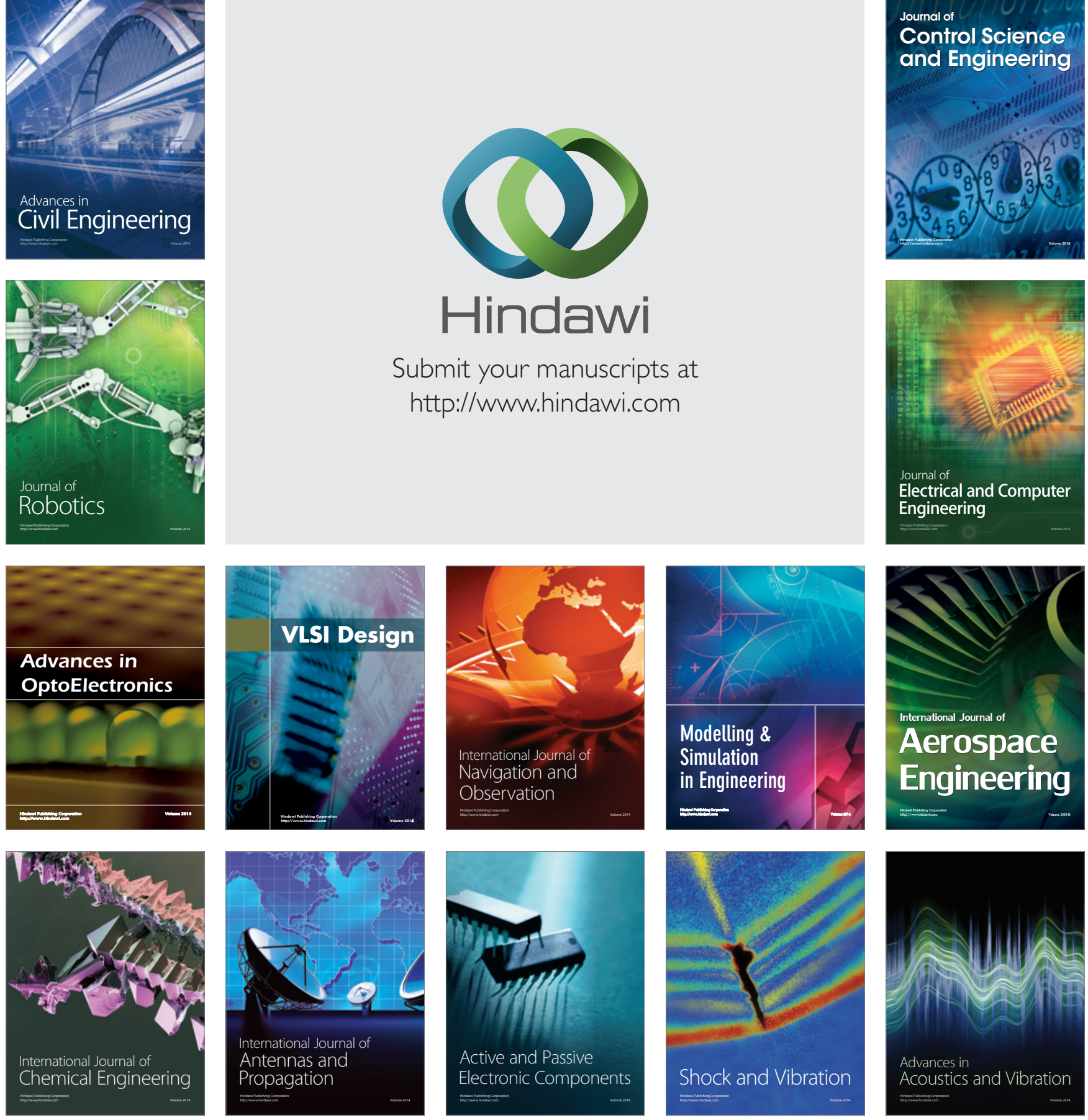